\title{
ACIDENTES DE TRANSITO : CARACTERIZAÇÃO DAS VITIMAS SEGUNDO O "REVISED TRAUMA SCORE" MEDIDO NO PERÍODO PRE-HOSPITALAR
}

\author{
MOTOR VEHICLE CRASH : VICTIMS' CHARACTERIZATION THROUGHOUT
}

\author{
"PREHOSPITAL REVISED TRAUMA SCORE"
}

\section{ACCIDENTES DE TRANSITO: CARACTERIZACIÓN DE LAS VÍCTIMAS SEGÚN EL}

\section{"REVISED TRAUMA SCORE" MEDIDO EN EL PERIODO PRE-HOSPITALARIO}

Marisa Amaro Malvestio* Regina Márcia Cardoso de Sousa**

Malvestio MA, Sousa RMC. Acidentes de trânsito: caracterização das vítimas segundo o "Revised Trauma Score" medido no período pré-hospitalar. Rev Esc Enferm USP 2002; 36(4): 394-401.

\section{RESUMO}

O estudo descreve idade, sexo, aspectos do mecanismo e procedimentos realizados em. 643 acidentados de trânsito atendidos nas Marginais Tietê e Pinheiros, considerando os valores do Revised Trauma Score (RTS) do periodo pré-hospitalar. As vitimas com $R T S=12$ somaram 90,8\%, com $R T S=11$, 4,0\% e RTS<10, 5,2\%. No grupo de RTS<10, destacam-se os atropelamentos (36,4\%), os impactos frontais (24,2\%), as vitimas projetadas (36,4\%) ou presas às ferragens (15,1\%), e que receberam o maior percentual de procedimentos de suporte avançado. Os motociclistas e as vitimas do gênero masculino e de idade entre 21 e 30 anos predominaram. Espera-se com este estudo, fornecer subsidios para a melhora da assistência às vitimas de acidente de trânsito.

PALAVRAS-CHAVE: Acidentes de trânsito. Revised Trauma Score. Atendimento de emergência pré-hospitalar.

\begin{abstract}
This report describes age, gender, trauma mechanics aspects and procedures from 643 motor vehicle crashes, MVC, victims in Tietê and Pinheiros expressways, by considering the prehospital Revised Trauma Score (RTS). The RTS=12 victims' were $90,8 \%$, with $R T S=11$ added $4,0 \%$ and in group with $R T S<10,5,2 \%$. Among the RTS $<10$ victims, the pedestrians stand out $(36,4 \%)$, the frontal impacts $(24,2 \%)$ and the projected $(36,4 \%)$ or trapped victims $(15,1 \%)$, and those that received advanced life support procedures.The motorcyclists and the male victims with 21 with 30 years of age were predominant. This study is expected to contribute to a better assistance to MVC victims.
\end{abstract}

KEYWORDS: Accidents, traffic. Revised Trauma Score. Emergency medical services.

\section{RESUMEN}

Este estudio tiene como objetivo describer, considerando el Revised Trauma Score (RTS) obtenido en el periodo pré hospitalario, edad, sexo, aspectos del mecanismo e procedimientos realizados en 643 víctimas de accidente de tránsito. Las víctimas con $R T S=12$ sumaron $90,8 \%$, con $R T S=11,4,0 \%$ y $R T S<10,5,2 \%$. De entre las víctimas con $R T S<10$, se destacan los atropellamientos (36,4\%), los impactos frontales (24,2\%) y las que fueron projectadas (36,4\%) y también aquellas que se encontraban presas entre los fierros $(15,1 \%)$. Los motociclistas y las víctimas de género masculino y de edad entre 21 e 30 años predominaron en todos las agrupaciones.

PALABRAS-CLAVE: Accidentes de tránsito. Revised Trauma Score. Servicios medicos de urgencia.

\footnotetext{
* Enfermeira. Gerente Operacional do Serviço de Suporte Avançado do Sistema Municipal de Atendimento Pré-hospitalar de São

Paulo. Doutoranda em Enfermagem pela EEUSP. E.mail: www.malvestio@dtlink.com.br

** Enfermeira. Livre docente Departamento de Enfermagem Médico-Cirúrgica da EEUSP. E.mail: www.vian@usp.br
} 


\section{INTRODUÇÃO}

A dimensão dos números envolvidos e a repercussão social, econômica e emocional fazem do trauma um dos maiores problemas deste final de milênio ${ }^{(1-3)}$. Dentre suas várias etiologias, o trauma envolvendo "veículo a motor", o chamado acidente de trânsito, tem forte impacto nas estatísticas de mortalidade por esta causa (4)

Além da potencialidade para altas taxas de mortalidade, os acidentes de trânsito têm em comum, a capacidade de provocar às vítimas um padrão de lesão semelhante" $(3,5)$

Este padrão de lesão e sua gravidade são influenciados pela aceleração, direção da força envolvida no acidente, a posição da vítima no veículo e sua idade, o uso de equipamentos de segurança, e ainda, tipo e tamanho dos veículos envolvidos (5). Todos estes fatores são relevantes na análise da biomecânica do evento e, conseqüentemente, influenciam a troca de energia entre os veículos e as pessoas envolvidas, além da gravidade do trauma e as intervenções necessárias.

No enfrentamento ao trauma, têm-se realizado intenso investimento no desenvolvimento de serviços de Atendimento pré-hospitalar, APH.

O Ministério da Saúde define APH, o "atendimento que procura chegar precocemente à vítima, após ter ocorrido um agravo a sua saúde, (de natureza traumática ou não traumática ou, ainda psiquiátrica), que possa levar a sofrimento ou mesmo a morte, sendo necessário, portanto, prestar-lhe atendimento e/ou transporte adequado a um serviço de saúde devidamente hierarquizado e integrado ao Sistema Único de Saúde ").

Este conceito prevê a chegada precoce a cena do acidente, de pessoal treinado e habilitado a executar a avaliação da vítima, os procedimentos de recuperação ou manutenção da vida e a decisão de triagem sobre a unidade hospitalar mais adequada para o atendimento definitivo.

É durante a fase de avaliação da vítima e decisão de triagem que o RTS torna-se uma ferramenta importante do $\mathrm{APH}$, permitindo à equipe, dimensionar a gravidade do trauma e empreender os recursos terapêuticos necessários à recuperação.

Para a análise da gravidade do trauma, na fase pré-hospitalar, tem sido freqüente a opção pelo uso de escalas fisiológicas de classificação(" e o Revised Trauma Score(8) é uma delas. Sua pontuação varia de 0 a 4 pontos para cada parâmetro: freqüência respiratória, a Escala de Coma de Glasgow e a pressão arterial sistólica, (valor máximo: 12 pontos). Quanto menores os valores, maiores as alterações nestes parâmetros e, portanto, a repercussão fisiológica provocada pelo trauma, caracterizando assim, sua gravidade (8).
A facilidade de aplicação, a rapidez na realização, a pequena exigência de treinamento e a aplicabilidade da escala durante o $\mathrm{APH}$, fizeram com que o seu uso, como indicador da gravidade e fator de decisão de triagem, se expandisse prioritariamente para esta fase do atendimento.

Neste sentido, os idealizadores da escala, buscaram determinar qual o valor de RTS que apresentava maior sensibilidade e maior especificidade na detecção de vítimas graves e que servisse como auxílio na decisão de triagem para transporte a um centro de trauma(8). Em seu estudo, os autores determinaram que :

- as vítimas de RTS =12 apresentaram parâmetros fisiológicos próximos dos normais, com discretas alterações fisiológicas, alcançando $99,5 \%$ de probabilidade de sobreviver (8)

- o valor RTS $\leq 11$ apresentou maior sensibilidade para as vítimas que, mesmo apresentando altos escores, e alta probabilidade de sobrevivência $(96,9 \%)$, possuíam lesões fechadas, cujos parâmetros fisiológicos ainda estavam em compensação (8).

- o valor de corte RTS $\leq 10$ foi o que maior especificidade apresentou, abrangendo o maior número de vítimas graves, com probabilidade de sobrevivência de $87,9 \%$ (8)

Baseados nestas considerações, ponderaram ser mais apropriado que as vítimas de RTS $\leq 11$, mesmo apresentando alta probabilidade de sobreviver, fossem levadas a centros de trauma para o atendimento definitivo(8).

Na cidade de São Paulo, o Serviço Municipal de $\mathrm{APH}$, modalidade Suporte Avançado à Vida, SAV, em convênio com a Companhia de Engenharia de Tráfego, instituiu um sistema de APH a acidentes de trânsito nas principais vias da cidade, a Marginal Tietê e a Marginal Pinheiros.

Consideradas "vias expressas" de trânsito, devido à velocidade permitida de até $90 \mathrm{~km} / \mathrm{h}$ e com um movimento diário de 1 milhão trezentos e cinqüenta mil carros em circulação, (um terço de toda a frota circulante da cidade de São Paulo), tais vias respondem pelo maior número de acidentes com vítimas na cidade ${ }^{(9-10)}$

Contando com equipes formadas por médicos e enfermeiros, o SAV utiliza o RTS como fator de decisão para triagem e indicador de gravidade das vitimas de acidentes de trânsito ocorridos nas referidas marginais.

Compreender a relação entre aspectos da biomecânica do acidente e a repercussão fisiológica provocada pelo trauma sofrido, favorece o conhecimento da população de vítimas atendidas, o que pode repercutir na melhora do cuidado, bem como, na adequação políticas públicas, tanto na esfera da saúde quanto na de trânsito. 


\section{OBJETIVO}

Descrever, considerando os valores iniciais do RTS, idade, sexo e aspectos relacionados com o mecanismo do trauma e procedimentos realizados nas vítimas de acidentes de trânsito, atendidas pelo Serviço Municipal de APH, modalidade Suporte Avançado à Vida.

\section{MÉTODO}

A análise quantitativa foi utilizada para caracterizar a população constituída de 643 vítimas de acidentes de trânsito, com idade entre 12 a 65 anos, cujos dados necessários estavam completos e, que foram atendidas pelo serviço municipal de APH, nas marginais Tietê e Pinheiros, no período de abril de 1999 a abril de 2000.

A fonte de informações foi a ficha de APH preenchida pelos médicos e enfermeiros da equipe, e os dados de interesse, foram registrados em um instrumento, sendo tratados nos programas Access e
Excel. Os resultados são apresentados em números absolutos e percentuais e em forma de tabelas ëe gráficos.

Dentre os dados registrados na ficha de APH, destacam-se o apontamento do RTSinicial,_ou seja, o valor do RTS alcançado pela vítima quando da chegada da equipe na cena, os aspectos do mecanismo do trauma como, tipo de veículo e posição da vítima no veículo, tipo de impacto sofrido, e localização da vítima, e ainda, os procedimentos realizados.

Para a análise proposta, as vitimas foram agrupadas, segundo o escore obtido no RTSiniciat, em : vítimas com RTSinicial igual a 12 (RTS=12); vítimas com RTSinicial igual a 11 (RTS=11), vitimas com RTSinicial igual ou menor que 10 (RTS $\leq 10)$.

\section{RESULTADOS E DISCUSSÃO}

O gráfico 1 demonstra que, das 643 vítimas deste estudo, 90,8\% das vitimas atendidas, apresentavam RTS $=12$, apenas 4,0\% igual a 11 e 5,2\% menor ou igual a 10 .

Gráfico 1 Distribuição das vítimas atendidas pelo SAV/192 segundo RTS inicial.São Paulo, Abril de 1999 à Abril de 2000.

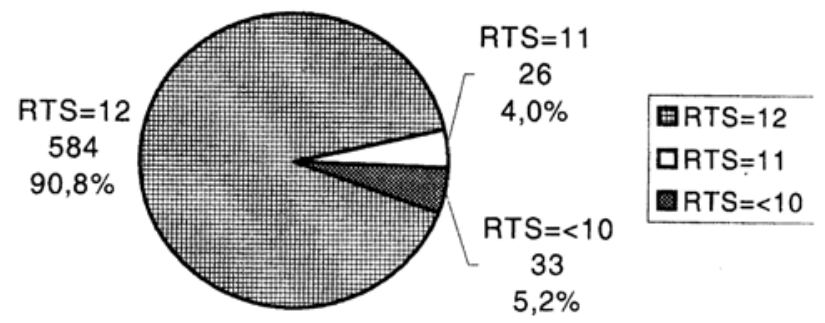

No presente estudo, o predomínio de vítimas com RTS $=12$, foi mais acentuado do que o apresentado por Bueno, Redeker, Norman(11), quando 68\% das vítimas estudadas obtiveram tal indice, ou Jacobs et al(7), que trabalhando com o Trauma Score(12) em populações de diferentes traumas, porém com maioria composta por acidentados de trânsito, encontraram escores máximos em 68\% .

Embora a maior velocidade permitida nas Marginais Tietê e Pinheiros, conferisse às vítimas deste estudo um potencial maior para apresentarem alterações fisiológicas acentuadas após um acidente, a adoção de medidas, como a Lei do uso de cinto de segurança e capacetes, o controle eletrônico de velocidade, e o novo Código de Trânsito Brasileiro, com rigorosas multas, certamente induziram os usuários destas vias a aderirem às leis. De trânsito(9-10), contribuindo para a diminuição dos indices de morbi-mortalidade, refletidos no alto percentual de vítimas que, neste estudo, permanecem com RTS =12, após o acidente.

Há de se considerar dois aspectos na comparação entre os estudos citados acima com relação a diferença entre os percentuais de casos de grande alteração fisiológica. O elevado volume de veículos nas Marginais Tietê e Pinheiros provoca grandes períodos de lentidão, onde a velocidade permanece baixa e, além disso, os estudos mencionados sobre análise de gravidade do trauma, utilizam dados intrahospitalares de instituições terciárias ou centros de trauma, para onde há triagem prévia de casos graves.

A tabela 1 apresenta a distribuição das vitimas, segundo suas características e agrupamentos de RTSinicial. 
Considerando o gênero, observa-se que as mulheres, estiveram em número menor nos 3 grupos analisados, e apenas uma apresentou RTS $\leq 10$. Roorda (13) demonstraram números semelhantes. A maior adesão ao Código de Trânsito bem como a medidas de direção defensiva, atos tipicamente femininos, pode estar contribuindo para a menor ocorrência de lesões graves que alterem os parâmetros fisiológicos, neste grupo de vítimas (9-10)

Considerando-se a idade, observa-se, na tabela 1 , que as vitimas com RTS igual a 12 e 11 concentramse nas faixas etárias até 39 anos. Entre as vítimas que apresentaram alteração fisiológica mais acentuada (RTS $\leq 10)$, destacam-se aquelas entre 21 a 30 anos, sendo que as com idade entre 39 e 48 anos também alcançaram percentuais expressivos $(21,2 \%)$, nesse grupo. Dentre as vitimas de maior faixa etária, todas do sexo masculino, encontram-se cinco casos de atropelamento e dois casos de pessoas presas em ferragens.

Foram, desta maneira, os adultos jovens, os mais presentes nos três grupos de vítimas, fato que permite inferências sobre os custos pessoais e sociais, pois compromete a qualidade de sobrevida e contribui para a diminuição de força de trabalho futuro.

Os ocupantes de motocicletas lideram, quando analisada a distribuição das vítimas por tipo de veículo, segundo o RTSinicial.

Quanto à posição da vítima no veículo, observase, na tabela 1 , percentuais próximos entre os condutores de motocicletas e motoristas com RTS $=11$ e RTS $\leq 10$. No caso de vítimas com RTS $=12$, a freqüência é menor para os motoristas $(24,1 \%)$, frente os condutores de motocicletas $(44,2 \%)$.

Embora os ocupantes de motocicleta não tenham a estrutura do veículo para protegê-los, absorvam toda a energia do impacto e comumente sejam jogados contra o chão, neste estudo, estes fatores não foram determinantes de maior freqüência de vítimas com alterações fisiológicas acentuadas, quando comparadas com os ocupantes de carro de passeio.

Quanto ao tipo de impacto, a tabela 1 demonstra que os atropelamentos predominam entre os quadros de maior alteração de parâmetros fisiológicos. Do total de vítimas com os mais baixos RTS, 36,4\% foram atropeladas. Ressalte-se, neste caso, o alto percentual de vítimas em que não foi possivel caracterizar o tipo de impacto , considerado sem informação , 18,2\%.

Segundo Hill(2) e Kong(3), o pedestre adulto recebe duplo impacto. O primeiro resulta em lesão das extremidades inferiores e o segundo compreende a cabeça e o tórax, devido à projeção contra o próprio veículo e, em seguida, contra o solo. Ainda, segundo estes autores, a velocidade do impacto, fator preponderante na avaliação da energia cinética, é o maior determinante da gravidade de lesão, pois, o pedestre, com sua massa relativamente pequena, não apresenta nenhuma resistência e absorve por completo a energia do impacto, o que eleva as taxas de morbimortalidade para este grupo de vítimas.

Enquanto, no agrupamento com RTS=12, a freqüência de vitimas de impacto frontal e lateral é bastante semelhante, nos grupos com escore de RTS inferior a 12, o impacto frontal supera o lateral.

Há indicativos, portanto, que o impacto frontal, com maior freqüência, provocou nas vítimas repercussões fisiológicas mais importantes do que o lateral. Ressalte-se, no entanto, que os impactos laterais ocorridos em vias com as características descritas neste estudo, não têm uma direção de força classicamente perpendicular(5), como no caso de impactos ocorridos em cruzamentos de ruas formando ângulos retos(5), e que seria responsável por um padrão de lesão específico, mas sim, formando um ângulo agudo, dado à mesma direção dos veículos envolvidos.

Rucholte(14) demonstraram, em seu estudo, que os mais graves acidentes foram os frontais, nas vítimas sem cinto de segurança, e que estas informações foram importantes na correlação com padrões de lesão específicos e expectativas de morbimortalidade.

Considerando-se a localização das vítimas, observa-se que, para os três agrupamentos de RTSinicial, prevaleceram aquelas que se encontravam fora do carro.

Parece óbvio que, após o acidente, a vítima, em boas condições físicas ou com lesões leves, consiga sair de seu veículo voluntariamente. A ação humanitária, mesmo que equivocada, de outros usuários que param para ajudar os acidentados, e como primeira medida, retiram as vítimas de dentro do veículo, também contribui para o alto percentual de vitimas que foram encontradas fora do veículo.

Ainda considerando a localização, é interessante observar o alto número de vítimas com RTS $\leq 10$, que foram projetadas ou se encontravam presas nas ferragens, respectivamente $36,4 \%$ e $15,1 \%$.

Dentre as vítimas projetadas com $\mathrm{RTS} \leq 10$, observou-se que cinco eram condutores de motocicletas, três foram atropeladas e duas eram motoristas, dentre outras que se encontravam na mesma condição.

As vítimas projetadas sofrem além do impacto decorrente do acidente, o impacto contra o solo, geralmente seguido de deslizamento. Considere-se, ainda, a possibilidade de terem sofrido um impacto intermediário contra o pára-brisa (nos casos de carros e caminhões) ou contra o guidão (no caso de bicicletas e motocicletas). Pode-se esperar destas vitimas maior probabilidade de lesões graves e portanto, maior repercussão fisiológica. 
A tabela 2 apresenta a distribuição das vítimas, segundo agrupamentos do RTSinicial e tipo de procedimento realizado.

Os procedimentos de imobilização foram os que apresentaram a freqüência mais constante e elevada em todos os agrupamentos de RTSinicial (entre $84,9 \%$ e $92,3 \%)$.

A imobilização, no APH, é um procedimentopadrão realizado tanto na presença de lesão ósteoarticular confirmada pelo exame clínico, quanto na suspeita baseada no mecanismo do trauma e na queixa

da vítima(1).

Esse procedimento-padrão compreende a imobilização da coluna cervical, com o uso de colar cervical em associação a prancha rígida, uso de modelos específicos de talas para imobilização de membros, e até do K.E.D., (Kendrick Extrication Device), equipamento próprio para retirada de vítimas do interior de veículos.
Outro fato notável na tabela 2 é o crescimento percentual da população que foi submetida a procedimentos de suporte respiratório e circulatório (básico e avançado), quanto mais baixo se apresentam os valores de RTS.

O gráfico 2 demonstra claramente este aspecto, encontrado também em estudos internacionais( ${ }^{(7-11)}$.

Conforme o gráfico 2 , o percentual de vítimas submetidas a procedimentos de acesso circulatório avançado atingiu a maior diferença entre os grupos de $\mathrm{RTS}=12$ e RTS $\leq 10(40,6 \%$ para $81,8 \%$, respectivamente), e os procedimentos de acesso respiratório básico e avançado foram os mais presentes nas vítimas de $\mathrm{RTS} \leq 10$.

O RTS pode ser considerado um excelente guia pré-hospitalar para a análise da resposta corporal à instalação do choque hipovolêmico hemorrágico, que é considerado um dos piores problemas dos politraumatizados $(1)$.

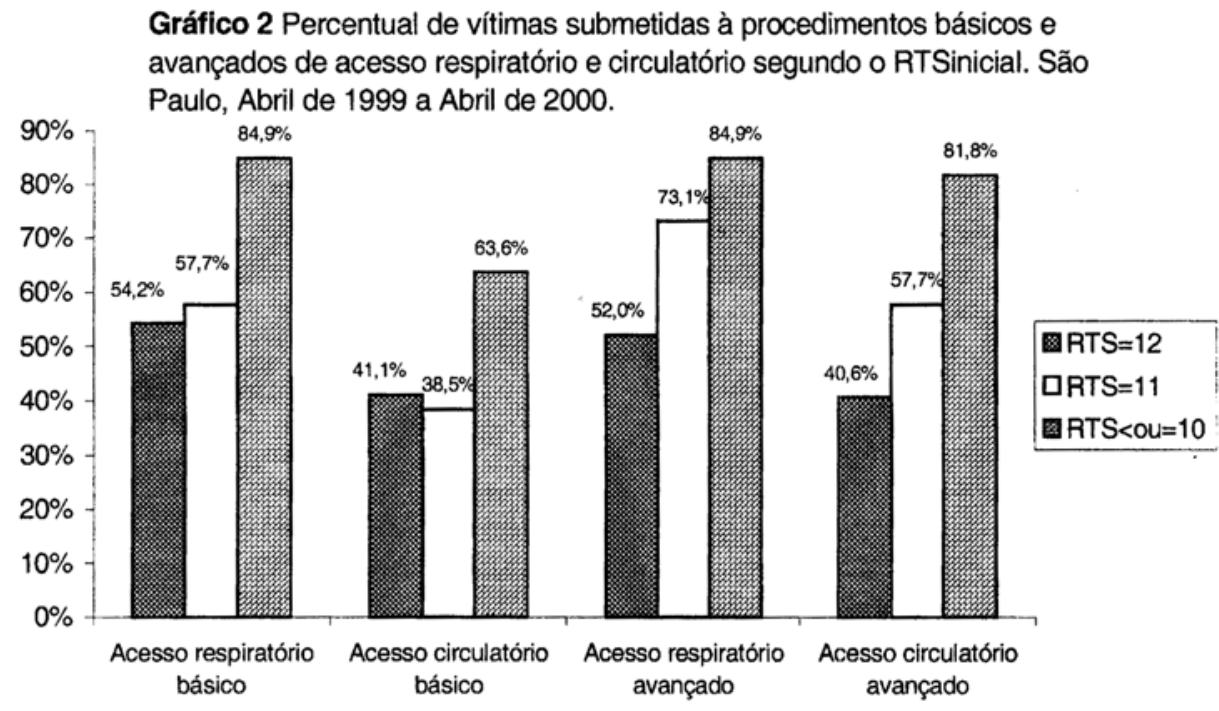

A oxigenioterapia e a punção venosa periférica com reposição de volume, utilizada principalmente com o objetivo de impedir ou reverter à instalação do choque hipovolêmico hemorrágico, nos pacientes de trauma, foram os procedimentos mais realizados nas vítimas de $\mathrm{RTS} \leq 10$. Claramente se observa, na tabela 2 que, quanto menor o RTS, maior a freqüência de punção venosa periférica com reposição de volume.

Em relação a oxigenioterapia, a mesma consideração anterior pode ser realizada, quando é constatado que este procedimento ocorreu isoladamente, dentro do acesso respiratório de suporte básico ou combinado com a utilização de Guedel ou aspiração.

Apesar da maior incidência destes procedimentos nas vítimas de RTS $\leq 10$, observa-se

RTS=12 apresentaram alta freqüência de realização de punção venosa periférica $(40,1 \%)$ e de oxigenioterapia $(54,2 \%)$. 0 potencial de risco das vítimas, sua instabilidade, a avaliação do mecanismo de trauma e a suposição de existência de lesões devido aos achados do exame físico, podem ter levado as equipes a adotar estes procedimentos como medidas preventivas ao agravamento do quadro, fato que encontra similaridade no estudo de Jacobs(7), onde $88 \%$ das vítimas foram submetidas à punção venosa, na cena do acidente, sendo apenas $45 \%$ nos escores mais baixos e o restante, nos altos escores.

A realização de curativos compressivos chama a atenção no grupo de procedimentos de acesso circulatório básico. Mantendo-se em um percentual de realização expressivo, dentro de todos os grupos 
de RTSinicial, este importante recurso da fase de APH auxilia na contensão de hemorragias externas, e portanto, na prevenção à instalação do choque hipovolêmico hemorrágico.

A compressão cardiaca externa, que caracteriza a realização de reanimação tardio-respiratória, foi um procedimento freqüente nas vitimas com RTS $\leq 10$, atingindo percentual de 33,3\%.

Segundo os protocolos de atendimento, as vítimas com valor $\leq 8$ na Escala de coma de Glasgow, devem receber intubação(1). Neste estudo, dentre as vítimas com RTS $\leq 10(\mathrm{~N}=33)$, encontrou-se 18 $(54,6 \%)$, que foram submetidas a intubação. No entanto, o percentual de realização deste procedimento, no total de vitimas, $(\mathrm{N}=643)$, é de $2,8 \%$.

Os resultados apresentados por Jacobs (7) quanto à realização de intubação em vítimas de diferentes traumas, demonstraram $16,2 \%$ dos atendimentos de SAV com este procedimento.

\section{CONCLUSÃo}

No estudo sobre a caracterização das 643 vítimas atendidas pelo SAV, segundo o RTS inicial, constatou-se que :

- A grande maioria dos acidentados apresentou RTS $=12$ (90,8\%), 4,0\% alcançou RTS $=11$ e 5,2\% obtiveram valores de RTS $\leq 10$.

- A predominância do sexo masculino se manteve dentre os agrupamentos, acentuando-se nos quadros de maior alteração de parâmetros. A liderança dos adultos jovens (de 21 a $<30$ anos) se deu de forma semelhante em todos os grupos .

- Quanto ao tipo de veículo e posição da vítima, a motocicleta e seu condutor lideraram em todos os agrupamentos de RTSinicial. Os carros a passeio e os motoristas compuseram o segundo grupo mais atingido.

- Os impactos frontais superaram os laterais dentro dos 3 grupos de RTSinicial, porém nos casos de RTS < 10 as diferenças percentuais foram mais expressivas, sendo possivel relacioná-los com os quadros de maior alteração de parâmetros

- O tipo mais representativo de impacto nas vitimas de RTS < 10 foi o atropelamento.
- Quanto à localização das vítimas, as projetadas e as que se encontravam presas às ferragens destacaramse no grupo dos apresentaram maior alteração de parâmetros, no entanto, prevaleceram as vítimas que se encontravam fora do veículo.

-Dentre todos os procedimentos, as imobilizações foram as mais realizadas em todos os agrupamentos de RTSinicial (entre 84,9 e 92,3\% das vítimas).

- Dentre os procedimentos de Suporte Básico Respiratório e circulatório, respectivamente a oxigenioterapia e o curativo compressivo foram os mais realizados.

- Quanto aos procedimentos avançados, a intubação só foi observada nos quadros de RTS $\leq 10$, sendo que a punção venosa periférica se apresentou, de forma expressiva, mesmo nos quadros de RTS $=12$ e $=11$, apresentando uma freqüência percentual de realização maior nos quadros com alterações fisiológicas mais importantes (RTS $\leq 10)$.

- No geral, quanto mais baixo o RTSinicial, maior o percentual de vitimas que foram submetidas a procedimentos.

Apesar da utilidade do uso de escores na cena do acidente por pessoal de APH, o uso exclusivo de seus parâmetros não é suficiente na determinação dos quadros graves que necessitam maior intervenção. Associá-los a informação sobre os mecanismos do trauma pode ser muito útil e tem sido incentivada $^{(1,4-5)}$ Esta atual e forte tendência vem se fortalecendo a medida que os serviços de APH são capazes de, organizadamente, colher estas informações, tornando possivel relacioná-las com as lesões e prognósticos, fornecendo subsídios à tomada de decisão na triagem e gerando um rico corpo de conhecimentos a cerca da vítima e do acidente.

Esse conhecimento detalhado da população de vítimas de acidente de trânsito pode repercutir na melhora do atendimento e no direcionamento do tratamento, tanto na fase pré quanto intra-hospitalar, além de subsidiar decisões estratégicas na gestão do trânsito e da saúde pública.

O fato de não terem sido avaliadas as lesões das vítimas, no presente estudo, é uma importante perspectiva para pesquisas complementares ao tema. 
Tabela 1- Distribuição das vitimas de acidente de trânsito atendidas pelo SAV, segundo o RTSinicial e características . Silo Paulo, Abril/1999 à

Abril/2000.

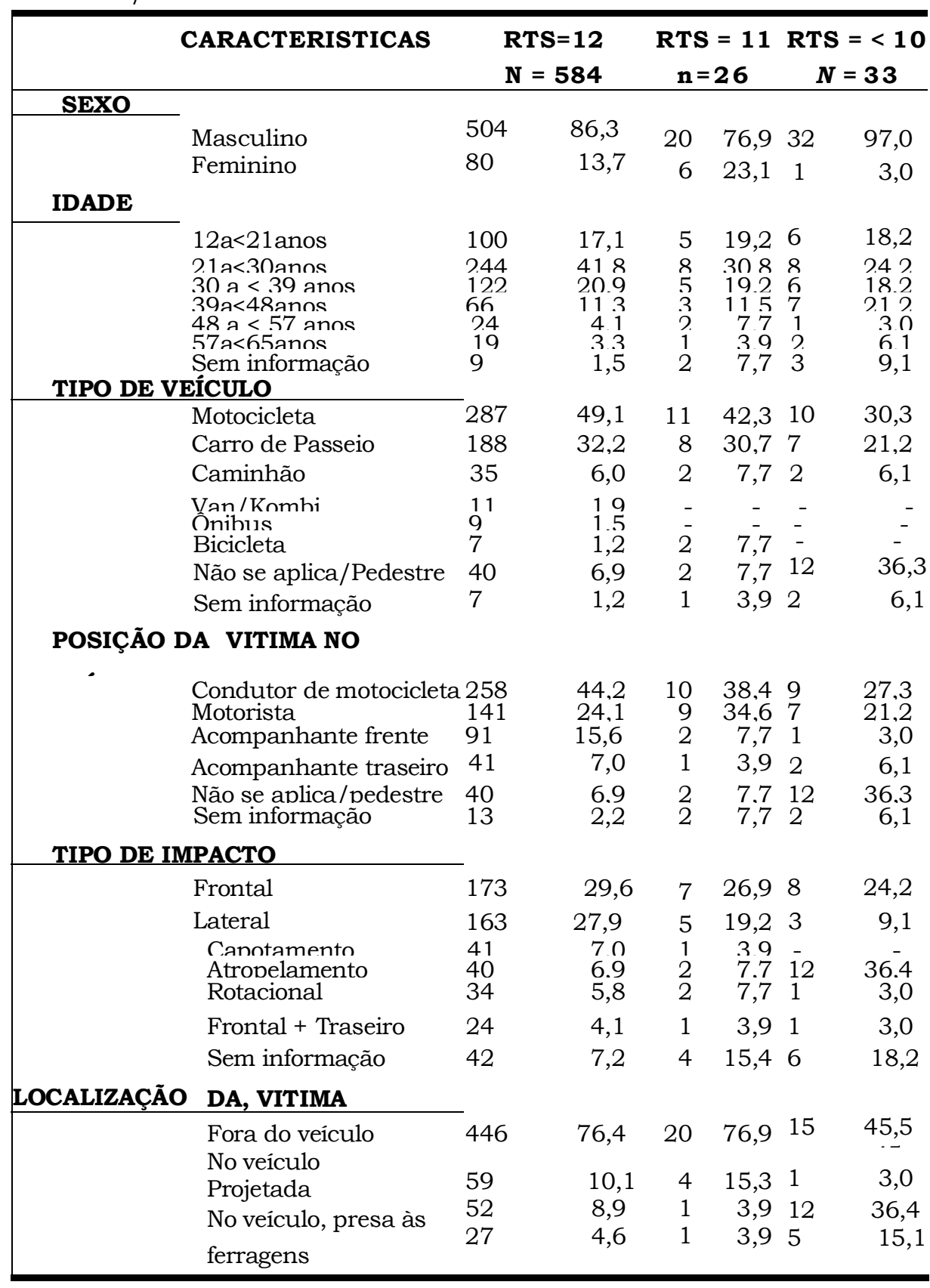

Tabela 2 - Distribuição das vítimas de acidentes de trânsito atendidas pelo $\mathrm{SAV}$, segundo o RTSinicial e tipo de procedimentos realizados. São Paulo, Abril/1999 à Abril/2000 .

\begin{tabular}{|c|c|c|c|c|c|c|}
\hline \multirow[b]{2}{*}{ Agessga_Rogilie Sunorte Básico } & \multicolumn{2}{|c|}{$\begin{array}{l}\text { RTS }=12 \\
\text { N }=584\end{array}$} & \multicolumn{2}{|c|}{$\begin{array}{l}\text { RTS }=11 \\
N=26\end{array}$} & \multicolumn{2}{|c|}{$\begin{array}{c}\text { RTS }<10 \\
N=33\end{array}$} \\
\hline & & & & & & \\
\hline Oxigenioterapia & 311 & 53,3 & 15 & 57,7 & 9 & 27,3 \\
\hline 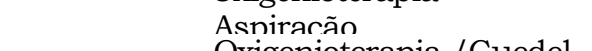 & - & - & - & - & 1 & 30 \\
\hline $\begin{array}{l}\text { Oxigeninterania / / iledel } \\
\text { Oxigenioterapia / Guedel }\end{array}$ & $\overline{5}$ & $\overline{0}, 9$ & $\begin{array}{l}- \\
-\end{array}$ & - & $\begin{array}{r}5 \\
13\end{array}$ & $\begin{array}{l}15.2 \\
39,4\end{array}$ \\
\hline $\begin{array}{l}\text { Realizado } \\
\text { Não Realizado }\end{array}$ & $\begin{array}{l}316 \\
268\end{array}$ & $\begin{array}{l}54,2 \\
45,8\end{array}$ & $\begin{array}{l}15 \\
11\end{array}$ & $\begin{array}{l}57,7 \\
42,3\end{array}$ & $\begin{array}{r}28 \\
5\end{array}$ & $\begin{array}{r}84,9 \\
1501\end{array}$ \\
\hline Acesso Circulatório de Suporte básico & & & & & & \\
\hline
\end{tabular}

$$
\begin{array}{ccccrrr}
\text { Compressão torácica externa } & - & - & - & - & 6 & 18,2 \\
\text { Cirativn comnressivo } & 2.40 & 41.1 & 10 & 38.5 & 10 & 30.3 \\
\text { Comnressãn torácica } & - & - & - & - & 5 & 15.1
\end{array}
$$$$
\text { Curativo compresivo }
$$$$
\text { Realizado }
$$$$
\begin{array}{llllll}
240 & 41,1 & 10 & 38,5 & 21 & 63,6
\end{array}
$$$$
\begin{array}{llllll}
344 & 58,9 & 16 & 61,5 & 12 & 36,4
\end{array}
$$

Acesso respiratório de Suporte Avancado

$$
\text { Intubaçào }
$$$$
\begin{aligned}
& \text { Oximetria } \\
& \text { Intıhacãn / Oximetria } \\
& \text { Cricotireoidostomia }
\end{aligned}
$$

$$
\text { Realizado }
$$$$
\text { Não Realizado }
$$

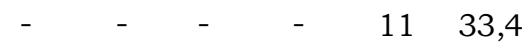
$\begin{array}{rrrrrr}30.3 & 52.0 & 19 & 7.3 .1 & 9 & 2.7 .3\end{array}$ \begin{tabular}{llllll}
\hline 03 & 52,0 & - & - & -1 & 3,0
\end{tabular} $\begin{array}{llllll}303 & 52,0 & 19 & 73,1 & 28 & 84,9\end{array}$ $\begin{array}{llllll}281 & 48,0 & 7 & 26,9 & 5 & 15,1\end{array}$

Acesso Circulatório de Suporte Avancado

Veia periférica com reposição volëmica

$\begin{array}{llllll}233 & 39,9 & 15 & 57,7 & 24 & 72,7\end{array}$ (VP)

$$
\begin{aligned}
& \text { Sutıura } \\
& \text { Monitoracãon } \\
& \text { VP/ Monitoração } \\
& \text { Realizado }
\end{aligned}
$$

$\begin{array}{llllll}- & - & - & - & - & - \\ 2 & 0.3 & - & - & - & - \\ 1 & 0.2 & - & - & 1 & 3.0 \\ 1 & 0.2 & - & - & 2 & 6.1\end{array}$ $\begin{array}{llllll}237 & 40,6 & 15 & 57,7 & 27 & 81,8\end{array}$ $\begin{array}{rrrrrr}347 & 59,4 & 11 & 42,3 & 6 & 18,2\end{array}$

\section{Imobilizac}

Não Realizado

$\begin{array}{crrrrrr}\text { Colar cervical + Membros + Prancha } & \mathbf{2 4 4} & 41,8 & 10 & 38,5 & 11 & 33,4 \\ \text { Colar cervical + Prancha longa } & \mathbf{2 3 6} & 40,4 & 13 & 50,0 & 16 & 48,5 \\ \text { Colar cervical + KED + Prancha } & \mathbf{3 2} & 5,5 & 1 & 3,8 & 1 & 3,0 \\ \text { Membros } & \mathbf{1 3} & 2,2 & - & - & - & - \\ \text { Realizado } & \mathbf{5 2 5} & \mathbf{8 9 , 9} & \mathbf{2 4} & \mathbf{9 2 , 3} & \mathbf{2 8} & \mathbf{8 4 , 9} \\ \text { Não Realizado } & \mathbf{5 9} & \mathbf{1 0 , 1} & \mathbf{2} & \mathbf{7 , 7} & \mathbf{5} & \mathbf{1 5 , 1}\end{array}$




\section{REFERÊNCIAS BIBLIOGRÁFICAS}

(1) Colégio Americano de Cirurgiões. Manual de suporte avançado a vida no trauma ATLS: programa para médicos. Rio de Janeiro 1996

(2) Hill DA, Delaney LM, Duflou J. A population-based study of outcome after injury to a car occupants and to pedestrians. J Trauma 1996; 40:351-5.

(3) Kong LB, Lekawa M, Navarro RA, McGrath J, Cohen M, Margulies DR et al. Pedestrian-motor vehicle trauma: an analy-sis of injury profiles by age. J Am Coll Surg; 1996:1723.

(4) Ministério da Sáude. Mortalidade Brasil-1995. Brasília; 1998.

(5) Hyde AS. Crash Injuries: How and why they happen. Florida: Hyde; 1992.

(6) Brasil. Portaria 2048 de 05 de novembro de 2002. Regulamenta os sistemas estaduais de urgência e emergência . Diário Oficial da República Federativa do Brasil, Brasilia 05 nov. 2002. Seção 1, p. 1 .

(7) Jacobs LM, Sinclair A, Beiser A, D'Agostino RB. Prehospital advanced life support : benefits in trauma. J
Champion. HR, Sacco WJ, Copes WS, Gann DS, Gennarelli $\mathrm{T}$, Flanagan ME. A revision of the trauma score. J Trauma 1989; 29:623-9.

(9) Secretaria Municipal de Transportes. Companhia de Engenharia de Tráfego de São Paulo. Fatos \& estatísticas de acidentes de trânsito 1997. São Paulo;1998.

(10) Secretaria Municipal de Transportes. Companhia de Engenharia de Tráfego de São Paulo. Sistema Integrado de Atendimento às emergências. São Paulo 1999.

(11) Bueno MM, Redeker N, Norman EM. Analysis of motor vehicle crash data in a urban trauma center : Implications for nursing practice and research. Heart Lung 1992; 21:558-67.

(12) Champion HR, Sacco WJ, Copes WS, Gann DS, Gennarelli T, Flanagan ME. Trauma score. J Trauma 1981; 9:672-6.

(13) Roorda J, Van Beeck EF, Stapert JWJL, Ten Wolde W. Evaluating perfomance of the revised trauma score as a triage instrument in the pre- hospital setting. Injury 1996; 27:163-7.

(14) Ruchholtz S, Nast-Kolb D, Waydhas C, Schweiberer L. The injury pattern in polytrauma: value of information regardind accident process in clinical acute management. Unfallchirurg 1996; 99:633-41. 